\title{
Study on the method and procedure of logistics system modeling and simulation
}

\author{
Hongbo WANG ${ }^{1,}$, , Zhongwei WANG ${ }^{2, b}$ \\ ${ }^{1}$ School of transportation and logistics, Central South University of Forestry and Technology, \\ Changsha, Hunan, China, 410004 \\ ${ }^{2}$ School of transportation and logistics, Central South University of Forestry and Technology, \\ Changsha, Hunan, China, 410004 \\ awanghongbocs@yahoo.com.cn, bwangpmp@163.com
}

Keywords: logistics system; modeling and simulation; simulation method; simulation procedure

\begin{abstract}
With logistics system becoming more and more complex, whose internal relationship becoming stronger than before, the modeling and simulation method also becomes more and more important during the process of logistics system completion and strategy. Simulation provides a new method for solving the complexity of logistics system. Through analyzing given logistics, constructing system model, setting input and output parameters, the right technology method suitable for the characteristics of logistics system can be found to make complete descript of logistics system. The problems in the present and future logistics system can be discussed and forecasted effectively, therefore the advice on optimizing logistics system can be provided to realize the logistics aim of low cost and high service level.
\end{abstract}

\section{Introduction}

With logistics system becoming more and more complex, whose internal relationship becoming stronger than before, the modeling and simulation method also becomes more and more important during the process of logistics system completion and strategy. Simulation is using computer to run model, imitate the situation of time system operation and the change process with time. Through the observation and statistics of the simulation operation process, the output parameters and basic characteristics of the simulated system are obtained to estimate the true parameters and performance. Simulation is the process of establishing real system model and conducting experience research by using the model.

Logistics system is a kind of complex discrete event system. In the process of system designing and controlling, there are many optimization problems. System simulation is an effective method to solve complex logistics system problem. Simulation is a good tool for designing and operating logistics system, which provides not only information for decision, but improves the decider's understanding level for the operation principles of logistics system. Simulation technology provides the optimal bonding point between technology and economics and the effective analysis method for complex logistics system planning.

\section{Logistics system introduction}

Logistics system refers to the organic integrity with specific function composed by some mutual-constraining and dynamic factors in a certain time and space, such as moving material, packaging equipment, handling machine, transportation tool, warehousing facilities, etc.

The aim of logistics system is to realize the time and space efficiency of material, to realize the links of logistics connected rationally, to get the optimal economic effect under the premise of protecting the process of social production.

A. The composition of logistics system

Logistics system is composed by transportation, storage, packaging, handling, moving, distribution, processing, information processing and some other subsystem[1]. Logistics operation 
system and logistics information system are the two main parts in logistics system. Logistics operation system is to make every kind of nodes networking to improve the efficiency of logistics activities. On the basis of protecting the smooth information flow in ordering, stocking, distributing, logistics information system is to make communication route and method networking to improve the efficiency of logistics operation system.

\section{B. The basic mode of logistics system}

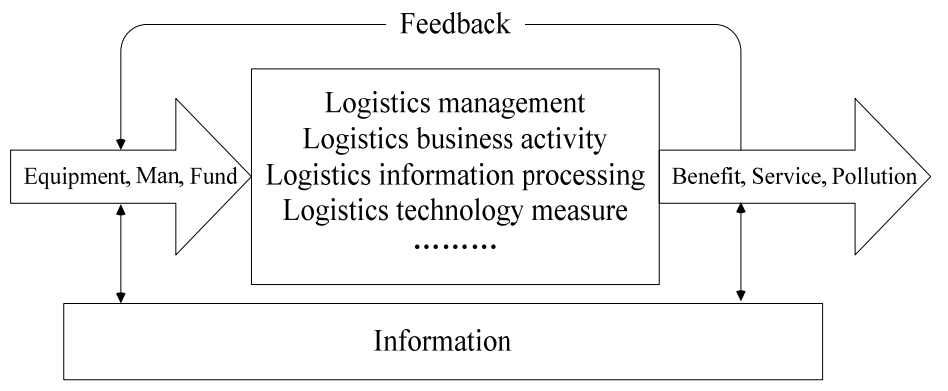

Fig.1 The basic mode of logistics system.

\section{The analysis content of logistics system}

(1) The external environment analysis of logistics system

Based on the situation of economy and technology at home and abroad, the position of logistics system in the external environment, national policy of logistics and some other factors are discussed, such as the distribution of resource and productive forces, logistics market and material, the level of production and technology.

(2) Internal analysis of logistics system

The content of the internal analysis of logistics system includes the characteristics of material demand change, demand quantity, demand object, demand composition and related demand connection method. The data of related logistics activities such as market distribution, purchasing channel, selling situation, and new technology, equipment, project of logistics production, and the conditions of stock and transportation are all included in the internal analysis.

\section{The concept of logistics system modeling and simulation}

\section{A. The characteristics of logistics system model}

Logistics system model is the abstract for real object and those useful and interesting features in real relationship, which is the description of the nature in logistics system[2]. Logistics system model description is a pro forma description for the logistics process in the real world. Model is a mathematical description for a certain profile. In a sense, model is a substitute for logistics system and simplicity also. At the same time, the model should be detailed to acquire effective conclusion of real logistics system from experiment. To construct model for real logistics system includes two tasks. One is to construct model's structure; the other is to provide data. When constructing model structure, it is necessary to make the border of logistics system clear, to identify the entity, attribute and activity of the logistics system. The data should make the relationship among attributes involved in the logistics activity certain. When selecting the model structure, there are two premises should be satisfied. One is the aim of model research should be detailed, the other is the relationship between specific modeling aim and the structure of logistics system should be known.

In general, the structure of logistics system model has the following features:

(1) Similarity logistics system model has similar characteristics and change rules with the targeted logistics system. It is the similar attribute and mathematical description between real logistics system and model.

(2) Simplicity from the perspective of practice, in the process of establishing logistics system model, the real model is a simplified similar model because of ignoring some subsidiary elements. Generally, the simpler the model is the better.

(3) Versatility to the logistics system composed by many entities, the different research aim decides the information related with logistics system is also different. So the model to indicate logistics system is not the sole. Because different analysts has different interest to logistics system or 
the same analyst want to understand the different change relations of logistics system, many kinds of models oriented to different research level is produced.

\section{B. The characteristics of logistics system simulation}

Logistics system simulation is the process of experiment research oriented real logistics system by using model or the process of simulating logistics system operation situation and law through establishing simulation model of real logistics system. Logistics system simulation has the following features:

(1) Logistics system simulation is oriented to practical process and systematical problems.

(2) Logistics system simulation technology is a kind of experiment method, which can acquire the knowledge of logistics system operation rules and features through computer. The difference between real logistics system experiment and simulated on is the simulation experiment is not dependent on the real logistics environment but the artificial one.

(3) Logistics system simulation research consists of many duplicated simulation processes, needs many times' experiments to estimate the performance and change rule of logistics system.

\section{Logistics system simulation}

The complexity of logistics system determines it is hard to describe it[3], even if the mathematical model is established. Computer simulation provides a new method for solving the problem. The complete descript of logistics system can be made through finding the right technology method suitable for the characteristics of logistics system.

\section{A. The types of logistics system simulation}

(1) The simulation of the flow in logistics system. There are many kinds of flow in logistics system, such as goods flow, vehicle flow, ship flow, financial flow, information flow, cash flow. Because of moving, dynamic simulation method should be adopted to describe the production, moving, disappearance, accumulation and transformation of flow.

(2) The simulation of queue in logistics system. Because the system composed by equipment and follow-up work belongs to queuing system, such as the running vehicle and station. The discrete simulation method is often used in this kind of simulation.

(3) The simulation of human factor in logistics system organization. Through computer simulation, thinking process of human being is described to provide better logistics system organization scheme.

\section{B. Logistics system simulation method}

(1) Continuous simulation method[4], it refers to the change of the system is smooth in time. This kind of method is used in the research on system development strategy, quantity forecast and some other continuous systems which has close relationship with time.

(2) Discrete simulation method[5], it refers to the change of the system is discrete in time. The activity causing the change is called as "event". Discrete simulation method mainly divided into event-based, activity-based, and process-based. The simulation model based on event is realized by defining the time change of event. The focus is the mechanism of time clock promoting and event scheduling. The simulation model based on activity is realized by describing the activities of system entity, setting beginning and ending conditions of activity in advance. This kind of model is suitable for the condition with variable activity time. The model based on process combined the features of the former two models, describing the process experienced in simulation time.

\section{The procedure of logistics system simulation}

To every simulation project, there are some specific procedures included. Whatever type the simulation project is, the basic process of simulation is constant[6], including the following steps:

(1) Defining problem, formulating object, describing system and assumed condition, listing the possible substitutive scheme, collecting data and information, establishing computer model, verifying and determining model, running model, analyzing output.

(2) Defining problem. A model can not describe all sides of the simulated reality system. Maybe the model describing all sides is too expensive or sometimes very bad, because of complexity and hardness in understanding. So the wise method is defining problem first, formulating object next, then constructing the model which can solve the problem completely. 
(3) Formulating object. The simulation research without object is useless. Object is the guide of all steps. The definition of the system is based on the object. It decides the assumption that should be made, the information should be collected. Of course the object should be definitive and practical.

(4) Describing system and assumed condition. Simply speaking, simulation model can reduce time to complete work. Time in logistics system can be divided into disposal time, transportation time and queuing time. System description includes resource, project route, project transformation, process control, producing time, resource breakdown time.

(5) Collecting data and information. Data can be got from history record, experience and calculation, which provides the basis for model input parameter and helps to collect some correct parameter data. The quantity of data demanded depends on the variance degree of variable, but there are some general rules. Then use this data to establishing computer model. During the course of constructing model, we need run and debug the model in every stage, then integrate them as a whole model. That is the true process of constructing model.

(6) Verifying and determining model. Verification is to confirm whether the function of model is coincident with the imagined function, whether dealing time and flow direction is right.

(7) Running model, analyzing output. Then the next step is to run model and analyze output. Through the step, we can get the output under different input parameters, and then the simulation outcome under different reality system is got.

\section{Conclusions}

Logistics system has the characteristics of complexity, multi-object, multi-level, which makes us meet so many problems when establishing multi-object function, even it is hard to establish by using mathematical model. Then there is a need for us to transform to utilize simulation technology to design logistics scheme. The utility of simulation technology in the whole logistics process can reduce the cost of designing logistics scheme and verifying it and help to realize the logistics aim of low cost and high service level. At present, logistics system simulation is applied in the following area: the simulation of automation stereoscopic warehouse, the simulation of stock system, vehicle scheduling simulation, the simulation of order processing in distribution center, etc. only by establishing corresponding model according to the feature of different logistics system, grasping the method that reflects the attributes of every feature, strictly following the procedure of simulation, can we make our model and simulation outcome valuable for the real logistics system.

\section{Acknowledgment}

Thinks for the support from the teaching research project of China Society of Logistics, The Ministry of education of higher school logistics professional teaching guidance committee (JZW2012124) and the support from the teaching research project of Central South University of Forestry and Technology (2011-15).

\section{References}

[1] Changbin Jiang, "Logistics system engineering”, Beijing: Electronic industry publishing house (2011).

[2] Lihua Guo and Mingyu Zhang, "The analysis of modern logistics system model of agriculture produc”, Economic problem, No. 6 (2006), pp. 53-54.

[3] Zhuwei feng and Fei Qi, “Simulation of Complex Logistics and It'S Research Status”, Journal of System Simulation, Vol. 15, No. 3 (2003), pp. 353-356.

[4] Yongchao Wang, "Virtual Simulation Modeling for Production Process of Continuous-discrete Hybrid Manufacturing System”, Journal of system simulation, Vol. 20, No. 9 (2008), pp. 2445-2448. 
[5] Qing Tian, Qingqing Xu, Ershi Qi, and Li Zheng, "Logistics system planning based on discrete manufacturing enterprise”, Industrial engineering, No. 6 (2006), pp. 52-54.

[6] Anglarti A, Grieco A, Pacella M, and Tolio T, “Object—oriented Modeling and Simulation of Flexible Manufacturing Systems: a Rule-based Procedure”, Simulation Modeling Practice and Theory, Vol. 10, No. 10 (2002), pp. 209-234.

[7] Lujiang xia, Fang Rong, and Lanxiu Ju, "Hot Research Areas of Simulation Technique in the Country--Review of Journal of System Simulation in recent years”, Journal of System Simulation, Vol. 16, No. 9 (2004), pp. 1910-1913. 